\title{
Performance Analysis of Bipolar Optical Code Division Multiple Access with Dual Electro- Optical Modulator Scheme for Free-Space Optical Communication
}

\author{
Eddy Wijanto ${ }^{1}$ \\ Department of Electrical Engineering, \\ Faculty of Engineering and Computer Science, \\ Universitas Kristen Krida Wacana, Jakarta \\ eddy.wiyanto@ukrida.ac.id
}

\begin{abstract}
In this study, the performance of bipolar optical code division multiple access (OCDMA) with dual electro-optical modulator (EOM) scheme for multi-user scenario was tested and analyzed with OptiSystem version 10. The performance measurement was implemented for free-space optical (FSO) communication and includes the common noises in optical communication. Two different channels were used in the measurement, i.e., additive white Gaussian noise (AWGN) and fading channel. Two extreme weather conditions, strong rain and storm, were considered in the simulation. The performance between three spectral amplitude coding (SAC) codes, i.e., Modified M-Sequence code, Walsh-Hadamard code, and random diagonal (RD) code were measured and compared. The simulation results indicate that Modified M-Sequence code had the highest BER while RD code achieved the lowest BER in the short-range and Walsh-Hadamard code got the lowest BER for the mediumrange of FSO, both for AWGN only and AWGN with fading channel. In strong turbulence condition, the performance of all codes become comparable after $500 \mathrm{~m}$ of FSO range. Modified M-Sequence suffered the lowest performance degradation while RD code endured the highest performance deterioration in all-weather condition. The results show that Modified M-Sequence can be applied for medium to long-range FSO.
\end{abstract}

Keywords: performance, SAC codes, bipolar OCDMA, dual EOM, FSO

\section{Introduction}

The requirements for the next generation network connectivity face some fundamental challenges that need to be addressed, some of them are higher system capacity, higher data rate, lower latency, improved quality of service (QoS), and higher security [1]. Further, the network beyond 5G demands the extreme reliability and availability with massive scalability and extreme mobility [2]. The network is projected to support a massive number of devices with ultra-low latency requirements to support the internet of everything and other near-future technology [3]. One of the prospective technologies to support these extreme requirements is optical communication. Besides the immunity to electromagnetic interference, optical communication enables long transmission distance to transmit massive amount of information with fast communication speed and relatively small delay. On the other hand, the complexity of 
fiber optic installation encourages the use of wireless optical communication, which transmits data via beams. One of the wireless optical communication technologies is freespace optical (FSO) communication. The FSO is license free with simple and fast installation at moderate cost. FSO has the ability to operate at higher power levels although the need of line of sight (LOS) condition limits its operation [4].

In order to support simultaneous multi-user transmission, multiplexing technology become one of important techniques in optical communication. The common multiplexing technology in optical communication is wavelength division multiplexing (WDM), which multiplexes a number of optical carrier signals by using different wavelengths of light [5]. The spectrum limitation motivates the researchers to improve the WDM by combining with orthogonal frequency division multiplexing (OFDM) technology [6]. Hybrid method between WDM and wireless networks was also proposed in [7]. Another multiplexing technique in optical communication is time division multiplexing (TDM) which improve the spectrum sharing by utilizing time slot between users. The optical clock distribution and synchronization are the main factor effecting the performance of the TDM [8]. Hybrid system was proposed to increase the performance of TDM [9]. A recent promising multiplexing technique is optical code-division multiple access (OCDMA). The advantages of OCDMA are the high-speed connectivity with asynchronous transmission, flexibility, security, and scalability. In order to relieve the impact of multi-user interference and phase-induced intensity noise (PIIN), spectral amplitude coding (SAC) with fixed in-phase cross-correlation was utilized [10].

Meanwhile, OCDMA can be categorized into non-coherent and coherent OCDMA. The non-coherent OCDMA system encode the optical signals by utilizing light intensity. The unipolar encoding is used in non-coherent OCDMA. This scheme is limited for low data rates application, such as voice communication. Further, coherent systems encode optical signals by using the input light phase. Bipolar encoding is commonly implemented in a coherent system [11]. With high spectral efficiency, bipolar coding is suitable for high-speed transmission and real-time applications. The Bipolar encoding outperform the unipolar encoding [12]. Several methods have been proposed to implement and improve the performance of bipolar encoding. Our previous work [13] implemented the switch for polarization coding in order to generate bipolar signals. The proposed scheme eliminates multiple access interference (MAI) in FSO systems. The limitation in the optical switch speed caused the limitation in transmission rate of the system [13]. In our further work [14], a dual electro-optical modulator (EOM) was proposed to improve the transmission rate. The results verified the visibility of the proposed scheme.

Furthermore, many different signature codes for SAC-OCDMA have been proposed. Three well-known SAC codes were implemented for the simulation in this paper, i.e., modified M-Sequence code [15], Walsh-Hadamard code [16], and RD code [17]. This study applied the bipolar OCDMA with dual EOM scheme in the multi-user scenario for performance measurement with several SAC codes. Two channel conditions were adopted, i.e., additive white Gaussian noise (AWGN) and AGWN with fading channel in the extreme weather conditions.

The remainder of this paper is organized as follows. Section 2 describes the bipolar OCDMA system with dual EOM used in this paper for performance measurement, including the system architecture of encoding and decoding scheme and the correlation function for MAI elimination. Section 3 presents the simulation setup for performance 
measurement, including the simulation parameters, noise, and channel model implemented in the simulation. Section 4 shows the results, including the performance measurement and comparison of the bipolar OCDMA systems with dual EOM in the extreme weather conditions, both for AWGN and fading channels, respectively. Finally, Section 5 conclude the work along with the proposed future work.

\section{Bipolar OCDMA System with Dual EOM}

The performance measurement in the simulation implemented the bipolar OCDMA with dual EOM scheme proposed in our previous work [14]. The performance of bipolar OCDMA with dual EOM scheme increase the transmission rate compare to switch scheme [13]. Instead of using fiber Bragg grating (FBG)-based encoding, in this simulation, continuous wave (CW) laser array was used for encoding in accordance with the signature code. The erbium-doped fiber amplifier (EDFA) was used as pre-amplifier.

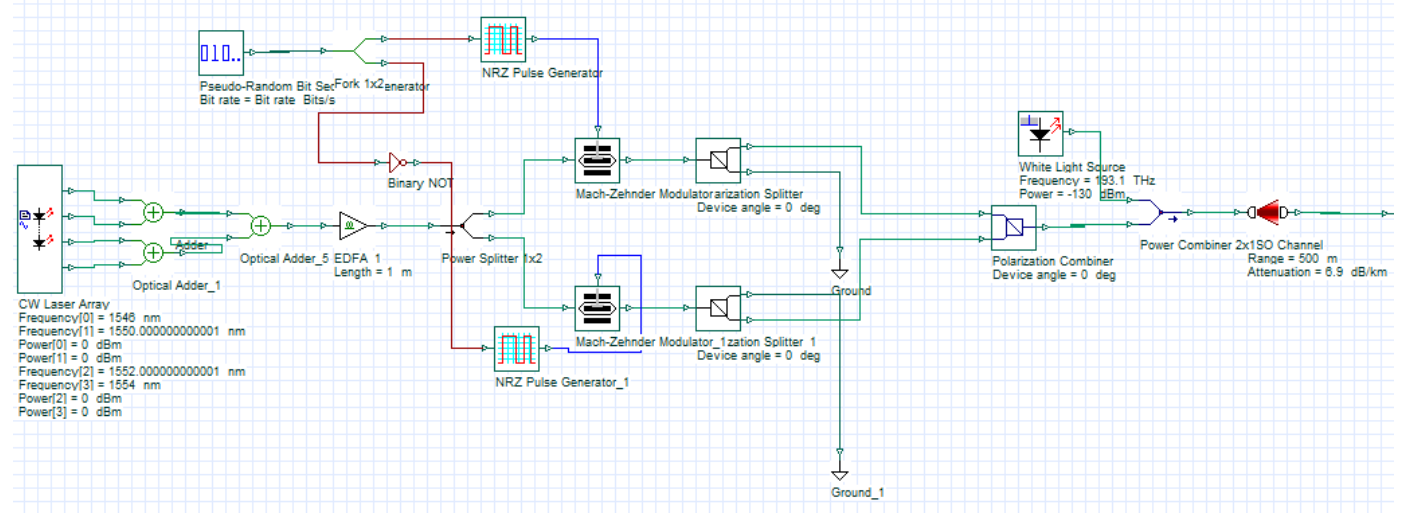

Figure 1. Bipolar OCDMA with dual EOM encoder scheme

First, the user information signal was modulated by EOM with CW laser array according to signature code. When the user information bit is " 0 " (or " 1 "), only lower (or upper) EOM will modulate the signal to the lower (or upper) polarization splitter. Lower (or upper) polarization splitter will polarize the optical signal to horizontal or vertical state. The polarized signal was further combined with polarization combiner before send to the FSO channel. Figure 1 depicts the encoder of the bipolar OCDMA with dual EOM used in this simulation.

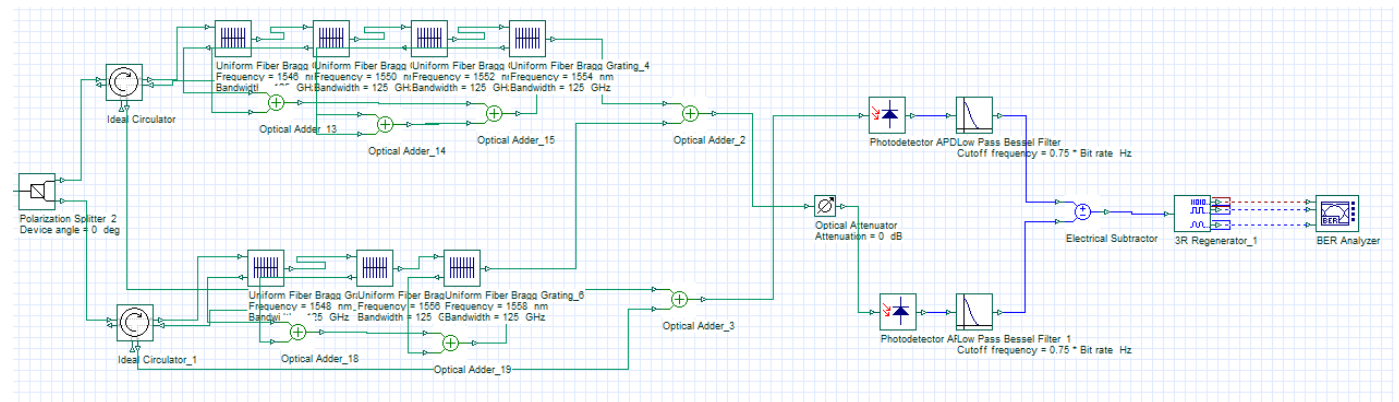

Figure 2. Bipolar OCDMA with dual EOM decoder scheme

After transmission through FSO channel, the optical signals were depolarized into horizontal and vertical state. The horizontal (or vertical) state will pas through lower (or 
upper) optical circulator port\#1. The optical signals from port\#2 of optical circulator further pass through the FBG decoder based on the signature code. The reflected spectra then gathered in the lower optical adder through the port\#3 of optical circulator while the transmitted signals from FBG will be gathered in the upper optical adder. After opticalelectrical signal conversion with avalanche photo-diode (APD), the upper and lower signals further subtracted, complete the balanced photo-detection. The BER and Q-factor were then measured using a BER analyzer. Figure 2 presents the decoder of the bipolar OCDMA with dual EOM used in this simulation.

For example, by using Walsh-Hadamard code as the signature code, when the user sends information bit of " 0 ", only lower EOM will modulate the signals to the lower polarization splitter. The signals further horizontally polarized and transmit through the FSO channel. In the receiver, the horizontally polarized signals will be depolarized into lower optical circulator. Since the lower optical circulator connect with complement FBG, the optical signals will be transmitted to the lower APD where there is no signal in the upper APD. The subtraction will be resulted in negative signals, represents the "- 1 " in the bipolar scheme. The same principle will work when user send information bit of " 1 ", the signals will appear in the upper APD where there is no signal in the lower APD. The subtraction will be resulted in positive signals, represents the " +1 " in the bipolar scheme. The multi-user scenario is expanded from single-user scenario by using the same design and principle. Figure 3 displays the example when user send alternate bit of " 0 " and "1" will resulted in " -1 " and " +1 " signals in the receiver-end.

Binary Sequence Visualizer

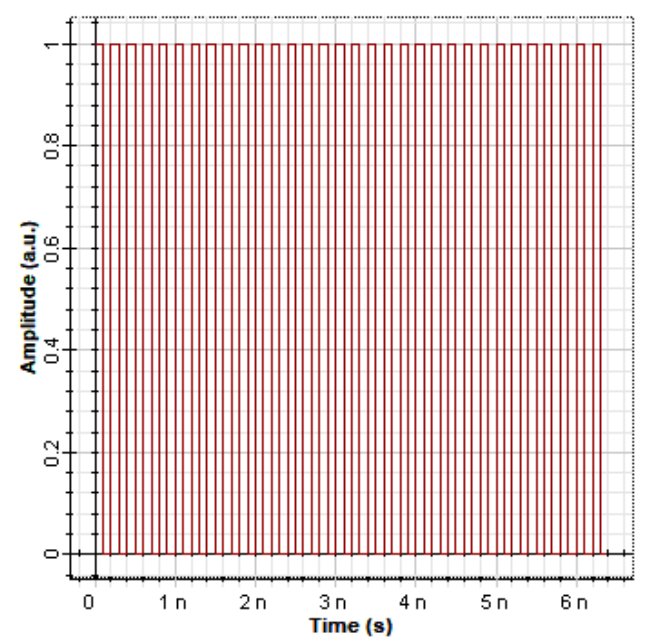

Oscilloscope Visualizer

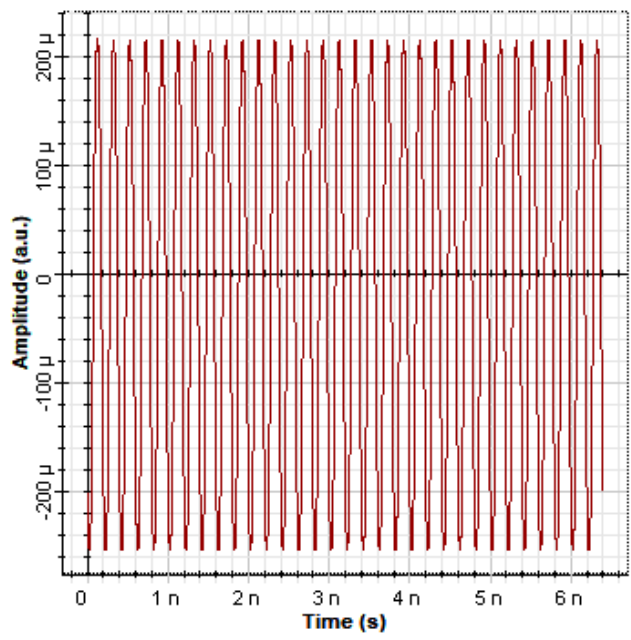

Figure 3. Input and output of bipolar OCDMA with dual EOM scheme

To implement the signature codes with length $N$ in the proposed schemes, the results of the following correlation must be obtained [14]:

$$
\begin{aligned}
& R_{X X}^{(n n)}(j, k)=\sum_{i=1}^{N} x_{j}^{(n)}(i) x_{k}^{(m)}(i)=\left\{\begin{array}{c}
(N) / 2, \text { for } j=k \text { and } n=m \\
(N) / 4, \text { for } j \neq k \text { and } n=m \\
0, \quad \text { otherwise }
\end{array}\right. \\
& R_{X X}^{(n m)}(j, \bar{k})=\sum_{i=1}^{N} x_{j}^{(n)}(i)\left[1-x_{k}^{(m)}(i)\right]=\left\{\begin{array}{c}
(N) / 4, \quad \text { for } j \neq k \text { and } n=m \\
0, \quad \text { otherwise }
\end{array}\right.
\end{aligned}
$$


where $n$ and $m$ denote the optical codewords with horizontal and vertical polarization states, respectively.

In order to verify the MAI elimination feature of the system, signature code of WalshHadamard code for user\#2 was used to decode the signals sent with signature code of Walsh-Hadamard code for user\#1. There are two signals appeared in the upper and lower APD after passing the FBG decoder, the subtraction process eliminates the signals. Figure 4 shows that nearly zero signals will be received in the decoding-end, proved the MAI elimination. The small signals come from the imperfect reflection of FBG decoders.

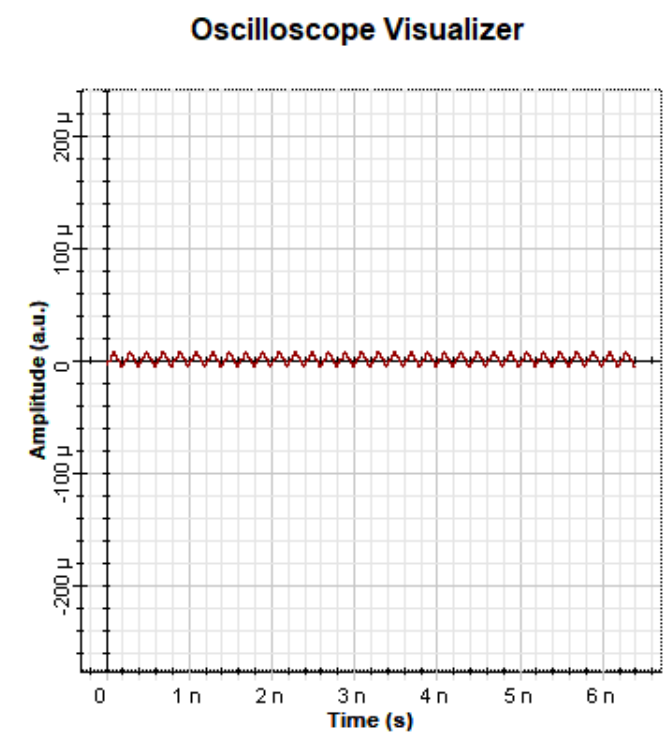

Figure 4. Received optical signals when user 2 signature code was used to decode the user\# 1 signature code

\section{Simulation Setup}

The performance analysis of bipolar OCDMA with dual EOM scheme was conducted in three simulations. First performance analysis was measured for AWGN channel with extreme weather conditions, i.e., strong rain and storm weather. Further simulation was conducted with AWGN and fading channel for extreme weather conditions, i.e., strong rain and storm weather. Final measurement was simulated for different turbulence level for storm weather condition. Table 1 shows the simulation parameters implemented in this study.

The performance measurement was analyzed with BER and Q-factor. The BER of the system can be calculated using [18]:

$$
B E R=\frac{1}{2} \operatorname{erfc}\left(\sqrt{\frac{S N R}{2}}\right)
$$

where $S N R$ is the signal-to-noise ratio of the proposed system and erfc denotes the complementary error function in time, which can be evaluated as [19]:

$$
\operatorname{erfc}=\frac{2}{\sqrt{\pi}} \int_{x}^{\infty} \exp \left(-t^{2}\right) d t
$$

In this simulation, for simplification of the analysis, the minimum log of the BER was used and can be derived as [20]:

$$
\min \{l \log (B E R)\}=\log _{10} B E R
$$


The correlation between the BER and Q-factor can be calculated as [21][22]:

$$
B E R=\frac{1}{2} \operatorname{erfc}\left(\frac{Q}{\sqrt{2}}\right)
$$

Table 1. Simulation parameters

\begin{tabular}{|c|c|c|}
\hline \multirow{4}{*}{ Global } & Parameter & Value \\
\cline { 2 - 3 } & Sample rate & $10 \mathrm{e}+009 \mathrm{bits} / \mathrm{s}$ \\
\cline { 2 - 3 } & Number of samples & $640 \mathrm{e}+009 \mathrm{~Hz}$ \\
\hline \multirow{4}{*}{ CW laser } & Linewidth & 4096 \\
\cline { 2 - 3 } & Power & $10 \mathrm{MHz}$ \\
\cline { 2 - 3 } & Azimuth & $0 \mathrm{dBm}$ \\
\hline \multirow{4}{*}{$\begin{array}{c}\text { Mach-Zehnder } \\
\text { Modulator }\end{array}$} & Wavelength & $1546 \ldots 1560 \mathrm{~nm}\left(\lambda_{1} \ldots \lambda 8\right)$ \\
\hline \multirow{3}{*}{\begin{tabular}{c} 
Uniform FBG \\
\cline { 2 - 3 }
\end{tabular}} & Extinction Ratio & $30 \mathrm{~dB}$ \\
\cline { 2 - 3 } & Symmetry Factor & -1 \\
\cline { 2 - 3 } & Bandwidth & $125 \mathrm{GHz}$ \\
\hline \multirow{4}{*}{$\begin{array}{c}\text { Avalanche } \\
\text { photodetector }\end{array}$} & Reflectivity & 0.99 \\
\cline { 2 - 3 } & Noise threshold & $-100 \mathrm{~dB}$ \\
\cline { 2 - 3 } & Gain & $1 \mathrm{~A} / \mathrm{W}$ \\
\cline { 2 - 3 } & Ionization ratio & 0.9 \\
\cline { 2 - 3 } & Dark current & $10 \mathrm{nA}$ \\
\cline { 2 - 3 } & Sample rate & $100 \mathrm{e}-024 \mathrm{~W} / \mathrm{Hz}$ \\
\hline \multirow{3}{*}{$\begin{array}{c}\text { FSO } \\
\text { channel }\end{array}$} & Thermal power density & $50-500 \mathrm{~m}$ \\
\cline { 2 - 3 } & Rransmitter aperture diameter & $5 \mathrm{~cm}$ \\
\cline { 2 - 3 } & Receiver aperture diameter & $20 \mathrm{~cm}$ \\
\cline { 2 - 3 } & Beam divergence & $2 \mathrm{mrad}$ \\
\hline
\end{tabular}

In order to measure the performance of bipolar scheme with closely real environment of the FSO system, some noises were included in the simulations, i.e., amplified spontaneous emission (ASE) noise, thermal noise, and shot noise with Gaussian distribution, where the total noise effect can be evaluated with [15]:

$$
\sigma_{\text {total }}^{2}=\sigma_{\text {th }}^{2}+\sigma_{\text {shot-S }}^{2}+\sigma_{\text {shot-ASE }}^{2}+\sigma_{S-A S E}^{2}+\sigma_{\text {ASE-ASE }}^{2}
$$

Each noise added in the simulation can be calculated as follows [15]:

- Thermal noise:

$$
\sigma_{t h}^{2}=\frac{4 \cdot k_{B} \cdot T}{R_{L}} \cdot E N B
$$

- Shot-Signal Noise:

$$
\sigma_{\text {shot }-S}^{2}=2 B_{e} \cdot\left(r M^{2} P_{S}+i_{d}\right)
$$

- Shot-ASE Noise:

$$
\sigma_{\text {shot }-A S E}^{2}=M^{2} F \cdot 2 B_{e} \cdot q r P_{A S E}
$$

- Signal-ASE Noise:

$$
\sigma_{S-A S E}^{2}=4 B_{e} \cdot r^{2} \cdot M^{2} \cdot P S D_{A S E} P_{s}
$$

- $\quad$ ASE-ASE Beat Noise:

$$
\sigma_{A S E-A S E}^{2}=r^{2} \cdot M^{2} \cdot P S D_{A S E}^{2}
$$

where $T$ is the absolute temperature of the device, $R_{L}$ denotes the receiver load resistance, $k_{B}$ is the Boltzmann constant, $E N B$ is the receiver equivalent electrical noise bandwidth, $B_{e}$ denotes the equivalent noise bandwidth of the photodetector, $r$ is the PIN 
responsivity, $M$ is the gain, $F$ denotes the excess noise factor, $P_{s}$ is the signal power, $i_{d}$ denotes the device dark current, $q$ is the electron charge value, $P_{A S E}$ is the optical noise power, and $P S D_{A S E}$ denotes the power spectral density of the optical noise field (spontaneous emission).

The simulation implemented the AWGN channel and fading channel caused by turbulence. The AWGN channel was simulated by adding a white light source with a power of $-130 \mathrm{dBm}$ to the optical signals before transmission through the FSO channel. The fading channel implemented the gamma-gamma distribution in this simulation with the probability of intensity $I$ can be evaluated with [15]:

$$
P(I)=\frac{2(\alpha \beta)^{(\alpha+\beta) / 2}}{\Gamma(\alpha) \Gamma(\beta)} I^{(\alpha+\beta) / 2-1} K_{\alpha-\beta}(2 \sqrt{\alpha \beta I})
$$

where $\Gamma(\ldots)$ is the gamma function, and $K_{\alpha-\beta}(\ldots)$ is the modified Bessel function of the second kind. $\frac{1}{\alpha}$ and $\frac{1}{\beta}$ are the variances of the small- and large-scale eddies, and can be observed as follows [15]:

$$
\begin{aligned}
& \alpha=\exp \left[\frac{0.49 \sigma_{R}^{2}}{\left(1+1.11 \sigma_{R}^{12 / 5}\right)^{5 / 6}}\right]-1 \\
& \beta=\exp \left[\frac{0.51 \sigma_{R}^{2}}{\left(1+0.69 \sigma_{R}^{12 / 5}\right)^{5 / 6}}\right]-1
\end{aligned}
$$

where $\sigma_{R}^{2}$ denotes the Rytov variance and is evaluated with [15]:

$$
\sigma_{R}^{2}=1.23 C_{n}^{2} k^{7 / 6} z^{11 / 6}
$$

where $C_{n}^{2}$ is the parameter index refraction structure with a value varying from $10^{-13} \mathrm{~m}^{-2 / 3}$ for strong turbulence to $10^{-17} \mathrm{~m}^{-2 / 3}$ for weak turbulence, $k$ is the optical wavenumber, and $z$ is the parameter range. In the simulation, $C_{n}^{2}$ was set to $10^{-13} \mathrm{~m}^{-2 / 3}$ for strong turbulence, $10^{-15} \mathrm{~m}^{-2 / 3}$ for medium turbulence, and $10^{-17} \mathrm{~m}^{-2 / 3}$ for weak turbulence.

Table 2. International visibility code [23]

\begin{tabular}{|c|c|c|c|}
\hline Weather Conditions & Precipitation & Visibility $(\mathrm{m})$ & Attenuation $(\mathrm{dB} / \mathrm{km})$ \\
\hline Light fog & Storm & 770 & 18.3 \\
\hline Very light fog & Strong rain & 1,900 & 6.9 \\
\hline Light mist & Average rain & 2,800 & 4.6 \\
\hline Very light mist & Light rain & 5,900 & 2 \\
\hline Clean air & Drizzle & 18,100 & 0.6 \\
\hline
\end{tabular}

Two extreme weather conditions were simulated based on the standard of propagation data required for the design of terrestrial free-space optical links from the International Telecommunication Union Radio Communication Sector (ITU-R) P.1817 [23]. The attenuations under different weather conditions are shown in Table 2.

Three SAC-OCDMA codes, that is, Modified M-Sequence code (15), WalshHadamard code, and RD code were applied in the simulation. These codes fulfil the correlation properties of Equation (1) and (2), indicated that the codes can be in the bipolar OCDMA with dual EOM scheme. The signature codes for two users used in this simulation are listed in Table 3. 
Table 3. Signature codes

\begin{tabular}{|c|c|}
\hline Code & Signature Code \\
\hline Modified M-Sequence & {$\left[\begin{array}{cccccccc}1 & 0 & 1 & 1 & 1 & 0 & 0 & 0 \\
1 & 1 & 1 & 0 & 0 & 1 & 0 & 0\end{array}\right]$} \\
\hline Walsh-Hadamard & {$\left[\begin{array}{llllllll}1 & 0 & 1 & 0 & 1 & 0 & 1 & 0 \\
1 & 1 & 0 & 0 & 1 & 1 & 0 & 0\end{array}\right]$} \\
\hline RD & {$\left[\begin{array}{cccccccc}1 & 0 & 0 & 1 & 0 & 1 & 1 & 0 \\
0 & 0 & 1 & 0 & 1 & 1 & 1 & 0\end{array}\right]$} \\
\hline
\end{tabular}

\section{Results and Discussion}

The first simulation implemented the multi-user bipolar OCDMA with dual electrooptical scheme for several different SAC codes with the AWGN channel condition. Two extreme weather conditions were conducted, i.e., strong rain and storm condition. The performance measurement was presented in terms of minimum log of BER and maximum Q-factor.

Figure 5 depicts the performance of SAC codes in the AWGN channel with strong rain condition. Figure 5(a) displays the minimum log of BER, where Modified MSequence code suffered the highest BER while RD code attained the lowest BER. All SAC codes maintained the acceptable BER, i.e., $10^{-9}$ for telecommunication and data communication. Figure 5(b) illustrates the maximum Q-factor, where RD code reached the highest Q-factor while Modified M-Sequence code endured the lowest Q-factor. All SAC codes maintained the acceptable Q-factor, i.e., 6 for telecommunication and data communication.

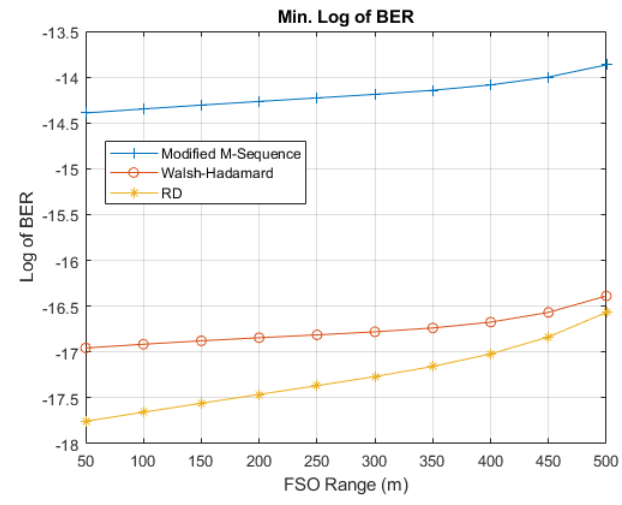

(a)

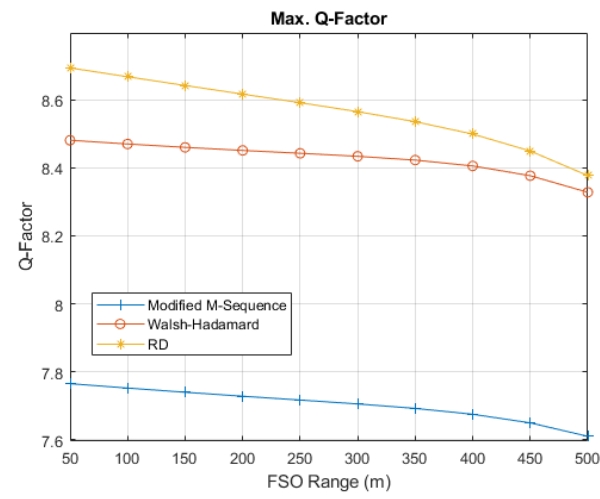

(b)

Figure 5. Performance of bipolar OCDMA in AWGN channel with strong rain condition (a) Min. log of BER (b) Max. Q-factor

Figure 6 indicates the performance of SAC codes in the AWGN channel with storm condition. Figure 6(a) shows the minimum log of BER, where Modified M-Sequence code suffered the highest BER. Up to $400 \mathrm{~m}$, RD code achieved the lowest BER and further outperformed by Walsh-Hadamard code after $400 \mathrm{~m}$. Until $500 \mathrm{~m}$, all codes maintained the acceptable BER, i.e., $10^{-9}$ for telecommunication and data communication. Figure 6(b) depicts the maximum Q-factor, where the lowest Q-factor was endured by Modified M- 
Sequence code. Up to $400 \mathrm{~m}$, the highest Q-factor was reached by RD code. After $400 \mathrm{~m}$, Walsh-Hadamard codes achieved the highest Q-factor. Up to $500 \mathrm{~m}$, all codes maintained the acceptable Q-factor, i.e., 6 for telecommunication and data communication.

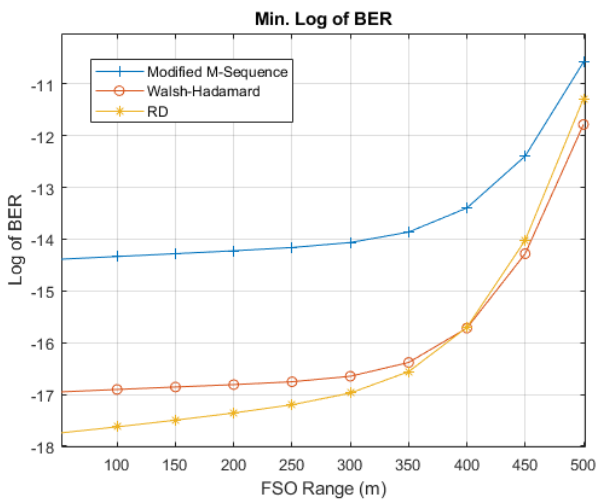

(a)

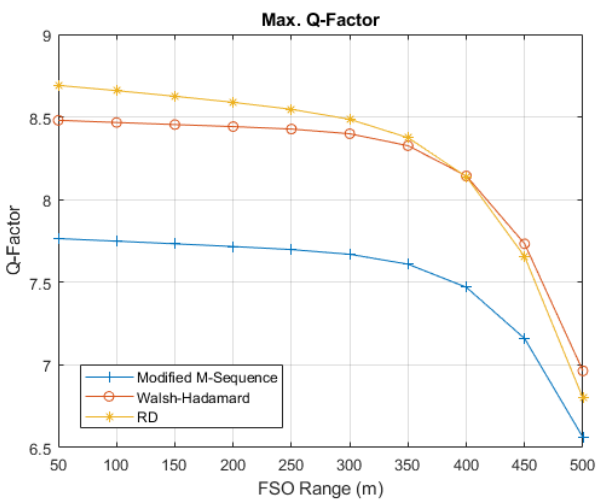

(b)

Figure 6. Performance of bipolar OCDMA in AWGN channel with storm condition (a) Min. log of BER (b) Max. Q-factor

Table 4 presents the performance degradation of SAC codes in AWGN channel for two extreme weather conditions. In strong rain weather condition, RD code suffered the highest performance deterioration while Modified M-Sequence code had the lowest performance degradation. Therefore, in the long-range of FSO, Walsh-Hadamard has the comparable performance with RD code. In storm weather condition, RD code suffered the highest performance degradation while Modified M-Sequence code had the lowest performance degradation. Hence, in the long-range of FSO, Walsh-Hadamard outperformed RD code performance in the storm weather condition.

Table 4. Performance degradation with the increasing of FSO range in AWGN channel

\begin{tabular}{|c|c|c|c|}
\hline Weather Condition & Code & Min. Log of BER (a.u.) & Max. Q-Factor (a.u.) \\
\hline \multirow{3}{*}{ Strong Rain } & Modified M-Sequence & 0.5254 & 0.15473 \\
\cline { 2 - 4 } & Walsh-Hadamard & 0.568 & 0.15288 \\
\cline { 2 - 4 } & RD & 1.1882 & 0.31648 \\
\hline \multirow{3}{*}{ Storm } & Modified M-Sequence & 3.8105 & 1.20244 \\
\cline { 2 - 4 } & Walsh-Hadamard & 5.1691 & 1.5158 \\
\cline { 2 - 4 } & RD & 6.462 & 1.89163 \\
\hline
\end{tabular}

The second simulation applied the multi-user OCDMA with dual EOM scheme for several different SAC codes with the AWGN and fading channel condition. Two extreme weather conditions were tested, i.e., strong rain and storm condition. The performance measurement was presented in terms of minimum log of BER and maximum Q-factor.

Figure 7 depicts the performance of SAC codes in the AWGN and fading channel with strong rain condition. Figure 7(a) displays the minimum log of BER, where Modified M-Sequence code suffered the highest BER while RD code attained the lowest BER. All SAC codes maintained the acceptable BER, i.e., $10^{-9}$ for telecommunication and data communication. After $500 \mathrm{~m}$ of FSO, both RD and Walsh-Hadamard code have a comparable performance. Figure 7(b) illustrates the maximum Q-factor, where RD code have the highest Q-factor while Modified M-Sequence code endured the lowest Q-factor. All SAC codes maintained the acceptable Q-factor, i.e., 6 for telecommunication and data 
communication. After $500 \mathrm{~m}$ of FSO, both RD and Walsh-Hadamard code have a comparable performance.

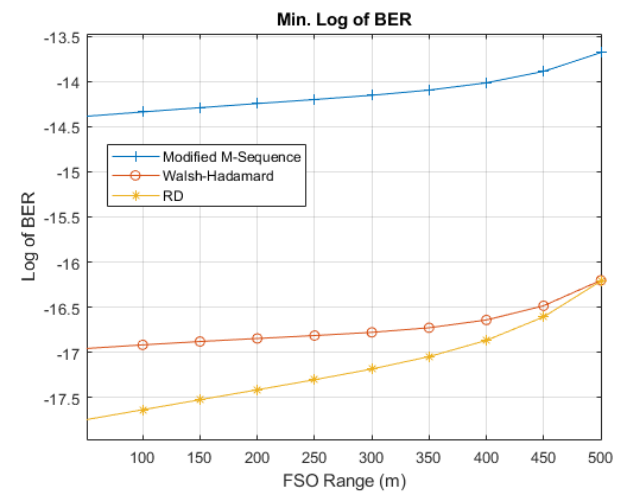

(a)

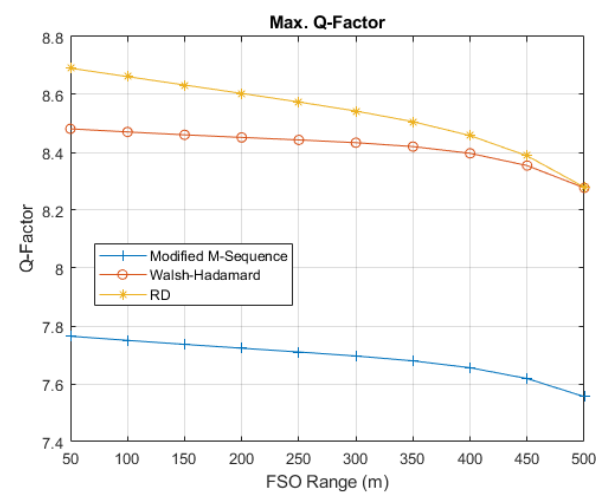

(b)

Figure 7. Performance of bipolar OCDMA in AWGN and fading channel with strong rain condition (a) Min. log of BER (b) Max. Q-factor

Figure 8 shows the performance of SAC codes in the AWGN and fading channel with storm condition. Figure 8(a) indicates the minimum log of BER, where Modified MSequence code endured the highest BER while RD code reached the lowest BER up to 350 m. After $350 \mathrm{~m}$, Walsh-Hadamard outperformed RD codes. Until $500 \mathrm{~m}$, all codes maintained the acceptable BER, i.e., $10^{-9}$ for telecommunication and data communication. Figure $8(\mathrm{~b})$ presents the maximum Q-factor, where the lowest Q-factor was suffered by Modified M-Sequence code and the highest BER was reached by RD code up to $350 \mathrm{~m}$. After $350 \mathrm{~m}$ of FSO range, Walsh-Hadamard code surpassed RD code performance. All codes maintained the acceptable Q-factor, i.e., 6 for telecommunication and data communication.

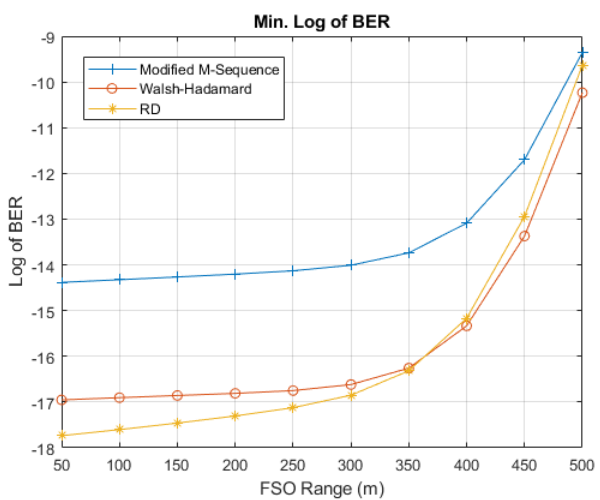

(a)

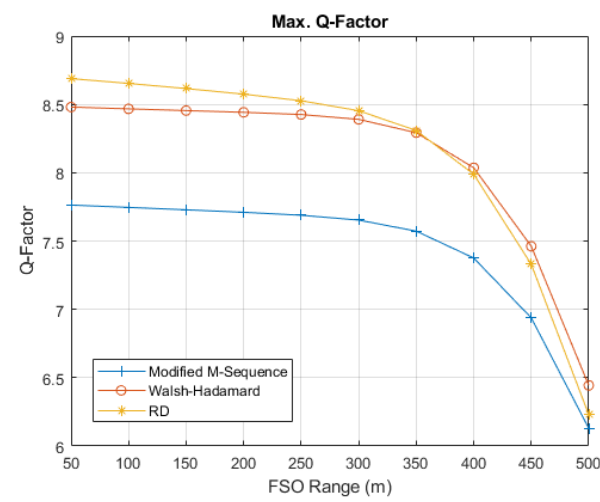

(b)

Figure 8. Performance of bipolar OCDMA in AWGN and fading channel with storm condition (a) Min. $\log$ of BER (b) Max. Q-factor

Table 5 shows the performance degradation of SAC codes in AWGN and fading channel for two extreme weather conditions. In strong rain weather condition, RD code suffered the highest performance degradation while Modified M-Sequence code had the lowest performance degradation. Hence, in the long-range of FSO, Walsh-Hadamard has the comparable performance with $\mathrm{RD}$ code. In storm weather condition, RD code 
suffered the highest performance deterioration while Modified M-Sequence code had the lowest performance degradation. Therefore, in the long-range of FSO, Walsh-Hadamard outperformed RD code performance in the storm weather condition.

Table 5. Performance degradation with the increasing of FSO range in AWGN and fading channel

\begin{tabular}{|c|c|c|c|}
\hline Weather Condition & Code & Min. Log of BER (a.u.) & Max. Q-Factor (a.u.) \\
\hline \multirow{3}{*}{ Strong Rain } & Modified M-Sequence & 0.7061 & 0.20871 \\
\cline { 2 - 4 } & Walsh-Hadamard & 0.7551 & 0.20395 \\
\cline { 2 - 4 } & RD & 1.5348 & 0.41118 \\
\hline \multirow{3}{*}{ Storm } & Modified M-Sequence & 5.02731 & 1.6342 \\
\cline { 2 - 4 } & Walsh-Hadamard & 6.718 & 2.03687 \\
\cline { 2 - 4 } & RD & 8.09886 & 2.45858 \\
\hline
\end{tabular}

The third simulation was used the bipolar OCDMA with dual EOM scheme for several different SAC codes with the AWGN and fading channel condition. Three different turbulence level with storm weather condition were implemented with multiuser scenario, i.e., weak, medium, and strong turbulence based on Equation (16). The performance measurement was presented in terms of minimum log of BER and maximum Q-factor.

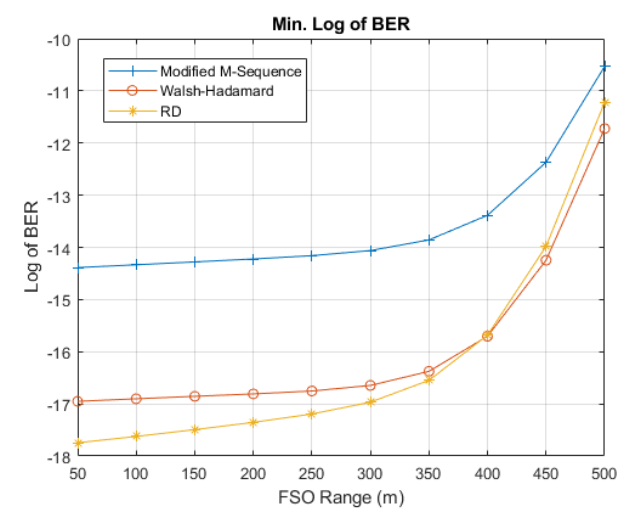

(a)

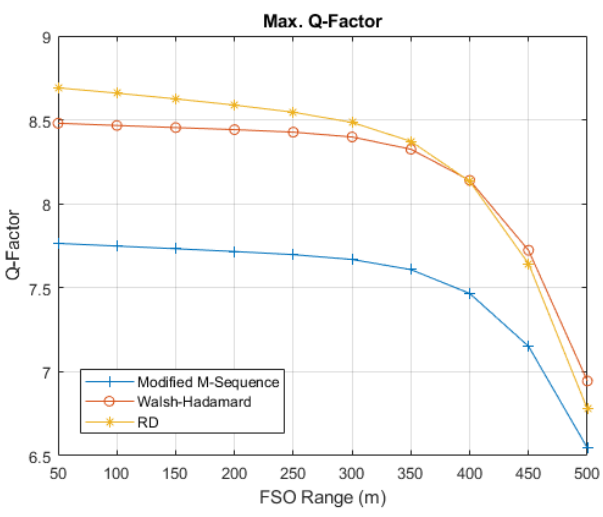

(b)

Figure 9. Performance of bipolar OCDMA in AWGN and fading channel with weak turbulence (a) Min. log of BER (b) Max. Q-factor

Figure 9 depicts the performance of SAC codes in the AWGN and fading channel for storm weather condition with weak turbulence. Figure 9(a) displays the minimum log of BER, where Modified M-Sequence code suffered the highest BER while RD code achieved the lowest BER up to $400 \mathrm{~m}$. After $400 \mathrm{~m}$ of FSO range, Walsh-Hadamard code outperformed RD code. Until $500 \mathrm{~m}$, all codes maintained the acceptable BER, i.e., 10-9 for telecommunication and data communication. Figure 9(b) illustrates the maximum Qfactor, where the lowest Q-factor was endured by Modified M-Sequence code and the highest Q-factor was achieved by RD code up to $400 \mathrm{~m}$. After $400 \mathrm{~m}$ of FSO range, WalshHadamard code surpassed RD code performance. Up to $500 \mathrm{~m}$, all codes maintained the acceptable Q-factor, i.e., 6 for telecommunication and data communication. 


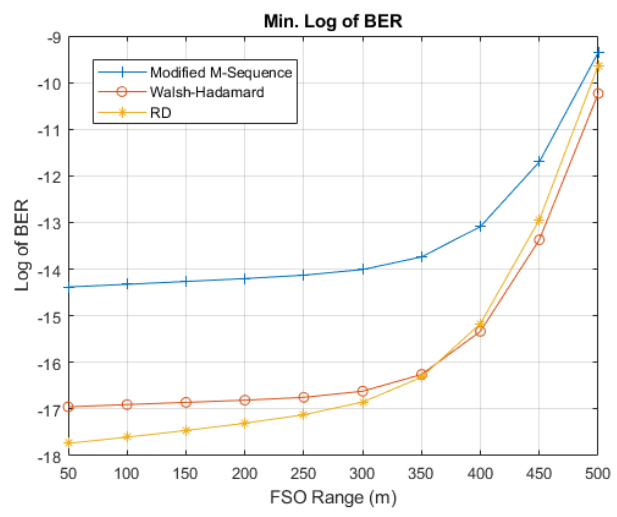

(a)

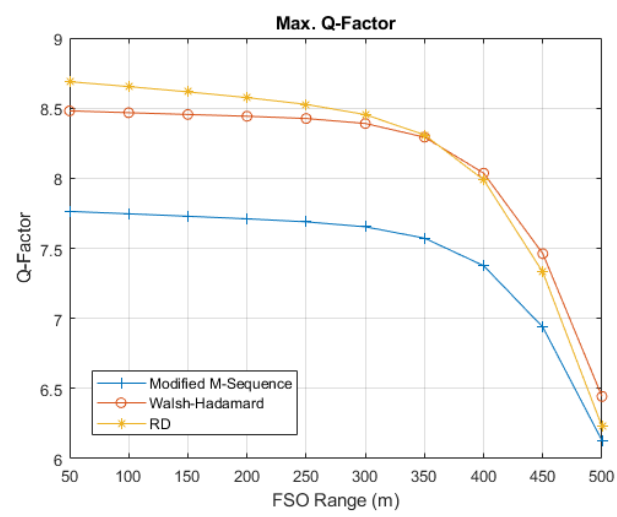

(b)

Figure 10. Performance of bipolar OCDMA in AWGN and fading channel with medium turbulence (a) Min. log of BER (b) Max. Q-factor

Figure 10 shows the performance of SAC codes in the AWGN and fading channel for storm weather condition with medium turbulence. Figure 10(a) presents the minimum $\log$ of BER, where Modified M-Sequence code suffered the highest BER while RD code had the lowest BER. Until $500 \mathrm{~m}$, all codes maintained the acceptable BER, i.e., $10^{-9}$ for telecommunication and data communication. After $350 \mathrm{~m}$ of FSO range, WalshHadamard code outperformed RD code. Figure 10(b) demonstrates the maximum Qfactor, where the lowest Q-factor was suffered by Modified M-Sequence code and the highest Q-factor was attained by RD code until $350 \mathrm{~m}$. After $350 \mathrm{~m}$ of FSO range, WalshHadamard code surpassed RD code performance. Up to $500 \mathrm{~m}$, all codes maintained the acceptable Q-factor, i.e., 6 for telecommunication and data communication.

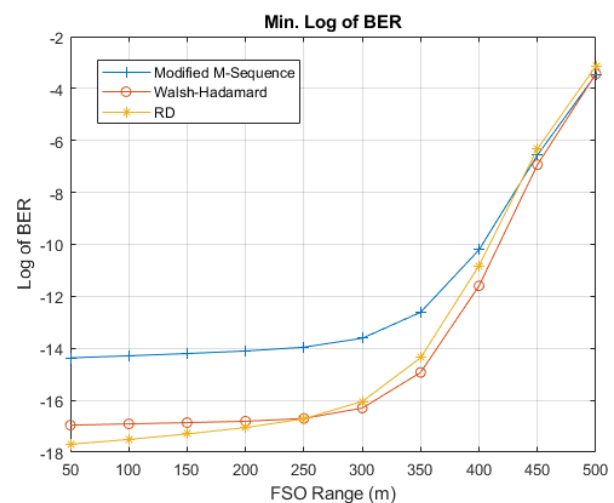

(a)

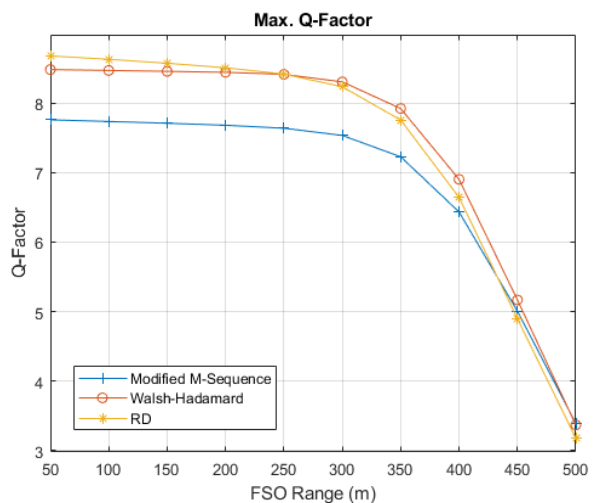

(b)

Figure 11. Performance of bipolar OCDMA in AWGN and fading channel with strong turbulence (a) Min. $\log$ of BER (b) Max. Q-factor

Figure 11 illustrates the performance of SAC codes in the AWGN and fading channel for storm weather condition with strong turbulence. Figure 11(a) displays the minimum $\log$ of BER, where Modified M-Sequence code endured the highest BER while RD code achieved the lowest BER up to $250 \mathrm{~m}$. After $250 \mathrm{~m}$, Walsh-Hadamard code outperformed the RD code. The performance of all codes become comparable after transmitted for 500 $\mathrm{m}$ of FSO. Up to $400 \mathrm{~m}$, all codes still achieved the acceptable BER, i.e., $10^{-9}$ for telecommunication and data communication. Figure 11(b) depicts the maximum Q-factor, where the highest Q-factor was achieved by RD code up to $250 \mathrm{~m}$. After $250 \mathrm{~m}$, Walsh- 
Hadamard outperformed RD code. Up to $400 \mathrm{~m}$, all codes maintained the acceptable Qfactor, i.e., 6 for telecommunication and data communication.

Table 6 presents the performance degradation of SAC codes in AWGN and fading channel for three different turbulence level. In weak turbulence, RD code suffered the highest performance degradation while Modified M-Sequence code achieved the lowest performance degradation. Therefore, in the long-range of FSO, Walsh-Hadamard outperformed RD code. In medium turbulence, RD code suffered the highest performance deterioration while Modified M-Sequence code had the lowest performance degradation. Hence, in the long-range of FSO, Walsh-Hadamard outperformed RD code performance. In strong turbulence, RD code suffered the highest performance deterioration while Modified M-Sequence code had the lowest performance degradation. Therefore, in the long-range of FSO, the performance of Modified M-Sequence is comparable with RD and Walsh-Hadamard code performance.

Table 6. Performance degradation with the increasing of FSO range in AWGN and fading channel

\begin{tabular}{|c|c|c|c|}
\hline Weather Condition & Code & Min. Log of BER (a.u.) & Max. Q-Factor (a.u.) \\
\hline \multirow{3}{*}{ Weak Turbulence } & Modified M-Sequence & 3.856 & 1.2181 \\
\cline { 2 - 4 } & Walsh-Hadamard & 5.2279 & 1.53488 \\
\cline { 2 - 4 } & RD & 6.5257 & 1.91284 \\
\hline \multirow{3}{*}{ Medium Turbulence } & Modified M-Sequence & 5.02731 & 1.6342 \\
\cline { 2 - 4 } & Walsh-Hadamard & 6.718 & 2.03687 \\
\cline { 2 - 4 } & RD & 8.09886 & 2.45858 \\
\hline Strong Turbulence & Modified M-Sequence & 10.90167 & 4.36503 \\
\cline { 2 - 4 } & Walsh-Hadamard & 13.52339 & 5.10897 \\
\cline { 2 - 4 } & RD & 14.55547 & 5.49727 \\
\hline
\end{tabular}

\section{Conclusion}

The bipolar OCDMA with dual EOM scheme can be implemented for several different SAC codes for multi-user scenario. The performance measurement shows that in the AWGN channel condition, Modified M-Sequence code suffered the highest BER. The lowest BER in AWGN channel condition in the short-range was achieved by RD code while Walsh-Hadamard code got the lowest BER for the medium-range of FSO. Meanwhile, for the AWGN and fading channel condition, Modified M-Sequence code suffered the highest BER. The lowest BER in AWGN and fading channel condition in the short-range was achieved by RD code while Walsh-Hadamard code reached the lowest BER for the medium-range of FSO. Similar performance results also measured in weak and medium turbulence, while in strong turbulence, the performance of all codes become comparable after $500 \mathrm{~m}$ of FSO range. For all of weather conditions, Modified MSequence suffered the lowest performance degradation while RD code endured the highest performance degradation. The results show that Modified M-Sequence can be applied for medium to long-range FSO. With the low performance degradation regarding the FSO length, Modified M-Sequence can achieve longer distance while maintaining minimum acceptable performance, especially in storm and strong turbulence condition. Future work can analyze the bipolar OCDMA scheme in high-order modulation system for multi-user scenario. 


\section{References}

[1] M. Chowdhury, Md. Shahjalal, S. Ahmed, Y.M. Jang, "6G wireless communication systems: applications, requirements, technologies, challenges, and research directions," IEEE OJ-COMS, pp. 1-1, 2020. Doi: 10.1109/OJCOMS.2020.3010270.

[2] C.D. Alwis, A. Kalla, Q.-V. Pham, P. Kumar, K. Dev, W.-J. Hwang, M. Liyanage, Madhusanka, "Survey on 6G frontiers: trends, applications, requirements, technologies and future research," IEEE OJ-COMS, vol. 2, pp. 836-886, 2021. Doi: 10.1109/OJCOMS.2021.3071496.

[3] M.W. Akhtar, S. Hassan, R. Ghaffar, H. Jung, S. Garg, M.S. Hossain, "The shift to 6G communications: vision and requirements," Hum. -centric comput. inf., vol. 10, 2021. Doi: 10.1186/s13673-020-00258-2.

[4] A. Malik, P. Singh, "Free space optics: current applications and future challenges," Int. J. Opt., vol. 2015, pp. 1-7, 2015. Doi: 10.1155/2015/945483.

[5] D. Ahlawat, P. Arora, S. Kumar, "Performance evaluation of proposed WDM optical link using EDFA and FBG combination," J. Opt. Commun., vol. 40, 2018. Doi: 10.1515/joc-2018-0044.

[6] A. Cardenas, V. Valderrama, B. Javier, "Performance of WDM-OFDM for using unequalized optical frequency comb," IEEE Lat. Am. Trans., vol. 13, pp. 2551-2558, 2015. Doi: 10.1109/TLA.2015.7331911.

[7] A.H. Ali, A. Desher, "Design and performance analysis of the WDM schemes for radio over fiber system with different fiber propagation losses," Fibers, vol. 7, no. 19, 2019. Doi: 10.3390/fib7030019.

[8] J. Zhang, A.B. Sharma, "High-speed optical time-division multiple-access (OTDMA) networks using optical signal processing," Photonic Netw. Commun. vol. 1, pp. 273-285, 1999. Doi: 10.1023/A:1010070632272.

[9] Z.J. Ren, K. Cui, J.X. Li, R.H. Zhu, Q. He, H.L. Wang, S.K. Deng, W.J. Peng, “Highquality hybrid TDM/DWDM-based fiber optic sensor array with extremely low crosstalk based on wavelength-cross-combination method," Opt. Express, vol. 25, no. 28870, 2017. Doi: 10.1364/OE.25.028870.

[10] C.B.M. Rashidi, S.A. Aljunid, F. Ghani, H.A. Fadhil, M.S. Anuar, "Phase induced intensity noise evasion in SAC-OCDMA systems using flexible cross correlation (FCC) code algorithm," Aust. J. Basic \& Appl. Sci, vol. 7, pp. 437-446, 2013.

[11] H.M.R. Al-Khafaji, S.A. Aljunid, H.A. Fadhil, "Spectral efficiency analysis of bipolar spectral amplitude coding optical code-division multiple access systems using different one-dimensional codes," IET Optoelectron., vol. 6, pp. 215-222, 2012. Doi: 10.1049/iet-opt.2011.0051.

[12] S.J. Park, B.K. Kim, B. Kim, "An OCDMA scheme to reduce multiple access interference and enhance performance for optical subscriber access networks," ETRI J, vol. 26, no. 1, pp. 13-20, 2004. Doi: 10.4218/etrij.04.0103.0002.

[13] H.C. Cheng, E. Wijanto, T.-C. Lien, P.-H. Lai, S.-P. Tseng, "Multiple access techniques for bipolar optical code division in wireless optical communications," IEEE Access, vol. 8, pp. 83511-83523, 2020. Doi: 10.1109/ACCESS.2020.2991071.

[14] S.P. Tseng, E. Wijanto, P.H. Lai, H.C. Cheng, "Bipolar optical code division multiple access techniques using a dual electro-optical modulator implemented in free-space optics communications," Sensors, vol. 20, no. 12, pp. 3583, 2020. Doi: 10.3390/s20123583. 
[15] E. Wijanto, C.-M. Huang, "Design of bipolar optical code-division multiple-access techniques using phase modulator for polarization coding in wireless optical communication," Appl. Sci., vol. 11, pp. 5955, 2021. Doi: 10.3390/app11135955.

[16] C.T. Yen, W. Chen, "A study of bipolar walsh-hadamard coding method in optical CDMA networks," Appl. Mech. Mater, no. 284-287, pp. 2667-2671, 2013. Doi: 10.4028/www.scientific.net/AMM.284-287.2667.

[17] H. Fadhil, S. Aljunid, R.B. Ahmad, "Performance of random diagonal code for OCDMA systems using new spectral direct detection technique," Opt. Fiber Technol., vol. 15, pp. 283-289, 2009. Doi: 10.1016/j.yofte.2008.12.005.

[18] H. Al-Khafaji, R. Ngah, S. Aljunid, T. Rahman, "A new two-code keying scheme for SAC-OCDMA systems enabling bipolar encoding," J Mod Opt, vol. 62, no. 5, pp. 327335, 2015. Doi: 10.1080/09500340.2014.978914.

[19] C.-T. Yen, H.-C. Cheng, Y.-T. Chang, W.-B. Chen, "Performance analysis of dual unipolar/bipolar spectral code in optical CDMA systems," J. Appl. Res. Technol., vol. 11, pp. 235-241, 2013. Doi: 10.1016/S1665-6423(13)71533-5.

[20] H. Mrabet, S. Mhatli, I. Dayoub, E. Giacoumidis, "Performance analysis of AOOFDM-CDMA with advanced 2D-hybrid coding for amplifier-free LR-PONs," IET Optoelectron., vol. 12, 2018. Doi: 10.1049/iet-opt.2018.5042.

[21] N. Dong-Nhat, M.A. Elsherif, A. Malekmohammadi, "Investigations of high-speed optical transmission systems employing absolute added correlative coding (AACC)," Opt. Fiber Technol., vol. 30, pp. 23-31, 2016.

[22] N. Avlonitis, E. Yeatman, M. Jones, A. Hadjifotiou, "Multilevel amplitude shift keying in dispersion uncompensated optical systems," IET Optoelectron., vol. 153, pp. 101-108, 2006. Doi: 10.1049/ip-opt:20050039.

[23] International Telecommunication Union. Propagation data required for the design of terrestrial free-space optical links, P Series Radiowave propagation, P.1817-1, 2012. 
